\title{
CYTOTOXIC AND ANTIVIRAL ACTIVITY OF Acacia catechu ON HUMAN PERIPHERAL BLOOD MONONUCLEAR CELLS
}

Department of Immunology
and Virology, Vidya
Pratishthan's School of
Biotechnology (VSBT,
Research centre affiliated to
Savitribai Phule Pune
University), Baramati,
District Pune, India. 413133

Submitted: $13-12-2015$

Revised: 25-02-2016

Accepted: 15-03-2016

${ }^{*}$ Corresponding author

Amit Gupta

Email:

amitgupta@vsbt.res.in

\section{Amit Gupta*, Sushama R Chaphalkar}

\begin{abstract}
As per the literature, medicinal plants showed enormous candidates who is responsible or showing antimicrobial, anticancer, antidiabetic, anti-inflammatory agents etc. Most of the research group focused only on those primary and secondary metabolites extracted from different medicinal plants and showed its antimicrobial (antiviral) activity against dreadful pathogens. The objective of our current study is to evaluate the cytotoxic and antiviral effect of aqueous leaves extract of Acacia catechu against new castle disease Virus (NDV) on human peripheral blood mononuclear cells (PBMC). For these studies, variable doses of aqueous leaves extract of Acacia catechu (0.5$30 \mathrm{mg} / \mathrm{mL}, 50 \mu \mathrm{L}$; dissolved in phosphate buffered saline, PBS) and examined its proliferation assay containing NDV and also determined CD14 monocyte surface marker in presence or absence of NDV using flow cytometry. The results showed that aqueous leaves extract of Acacia catechu inhibited NDV proliferation and also decline in CD14 monocyte surface marker with or without NDV at higher doses. Overall, aqueous extract of Acacia catechu at higher doses showed cytotoxic as well as antiviral effect and might be used for this purpose.
\end{abstract}

Key words: Acacia catechu; NDV; aqueous extract

\section{INTRODUCTION}

According to Ayurveda, medicinal plant products especially leaves, root, stem bark, flowers and seeds have been continuously used for the last so many years to treat number of animal and human diseases (Gupta et al., 2014; Maciel et al., 2002). Recently, number of medicinal plant products e.g. Aqadirachta indica, Mimusops elengi, Calotropis gigantea etc showed antimicrobial activity against specific bacterial/ viral/fungal disease (Maithani et al., 2011; Gupta et al., 2014; Patil et al., 2014). To further explore the knowledge of medicinal plant products, researchers focused only on those active constituent extracted from various primary or secondary metabolites which is responsible for inducing antimicrobial (viral/bacterial) activity against specific diseases (Gupta et al., 2014). These active metabolites could be isolated from the crude form of medicinal plant products and tested through various qualitative and quantitative based parameters i.e. TLC (thin layer chromatography); HPTLC (high performance thin layer chromatography) etc. Thus, the present work was totally focused on
Acacia catechu with emphasis on its anti-viral properties in human peripheral blood mononuclear cells (PBMC).

One of poultry infectious diseases i.e. new castle disease virus (NDV, single-stranded RNA virus; family Paramyxoviridae) is reported worldwide with several consequences including an increase in mortality rates (Gupta et al., 2014). Recently, treatments are not available for new castle disease virus however, vaccination is an effective method to control this disease but it does not provide any hundred percent protections. Thus there is a need to search for those medicinal plant products which restricted the load of NDV disease in poultry animals.

Acacia catechu (khair, family Fabaceae), medicinal plant and is popularly known for its astringent and antioxidant activities. The extracts of Acacia catechu exhibit various immunopharmacological effects like antipyretic, antidiarrhoeal, hypoglycemic, hepatoprotective; antioxidant activities etc (Lakshmi and Rajendran, 2012; Ray et al., 2006; Guleria et al., 2011). The current study was focused only on 
the immunopharmacological activity of Acacia catechu on human PBMC against NDV.

\section{MATERIALS AND METHODS Collection and preparation of plant material}

Fresh leaves of Acacia catechu was collected in the morning from Vidya Pratishthan's garden in the month of October 2015 in Baramati region, District Pune, Maharashtra, India. After collection of fresh plant leaves, first of all washed in running tap water in order to remove the dust particles and then with distilled water and finally dried in a shady area at room temperature. Afterwards, leaves were macerated with liquid nitrogen to prepare fine powder and were used for subsequent immunopharmacological assays.

\section{Qualitative and quantitative estimation of metabolites}

Secondary metabolites were determined qualitatively in the aqueous leaves extract of Acacia catechu. Different assays were performed qualitatively and revealed the presence of flavonoids (alkaline reagent test); terpenoids (acetic anhydride test) and phenolic compounds (ferric chloride test).

\section{Collection of NDV samples}

Poultry suspected disease samples of NDV were collected from suspected birds and these samples were studied under Biovillage programme scheme, Vidya Pratishthan's School of Biotechnology, Baramati, India. For these studies, pathogen free chicken eggs were used and these eggs were purchased from Venkys India Ltd for segregation and propagation of NDV from field samples using allantoic cavity route of embryonated (9-11 day old) chicken eggs (Gupta et al., 2014). These eggs were continuously observed in dark at regular time intervals, only bigger sized embryos were selected for inoculation. Embryo motility was observed continuously every $4 \mathrm{~h}$ through candling after insertion of supernatant $(200 \mu \mathrm{L})$ at $45 \square$ angle into embryonated chicken eggs. After the death of embryos, amnio-allantoic fluid was harvested and identified the presence of virus which is determined through haemagglutination (HA, 128) titre.

\section{Cytotoxicity and PBMC proliferation assay using NDV}

Anti-coagulant EDTA human blood samples were collected from Mangal Pathology Laboratory, Maharashtra, India and separated PBMC by means of density gradient centrifugation and then cultured for $48 \mathrm{~h}$ in 96 well plates with variable doses of aqueous leaves extract $(0.5-30 \mathrm{mg} / \mathrm{mL}, 50 \mu \mathrm{L})$ of Acacia catechu along with or without NDV (1:80 dilution, $10 \mu \mathrm{L})$. Thereafter, centrifuging the plates ( 96 well) at $1800 \mathrm{rpm}$ for $8 \mathrm{~min}$ at $4^{\circ} \mathrm{C}$ and add fresh complete medium into the 96-well plates. Again, incubating the plates for another $4 \mathrm{~h}$ along with MTT $(5 \mathrm{mg} / \mathrm{mL}, 10 \mu \mathrm{L})$. Afterwards, plates were centrifuged with discarding the supernatant, collecting the pellet and finally dispersing or dissolved in dimethyl sulphoxide (DMSO) solution. The optical density was measured at 570nm (Gupta and Chaphalkar, 2016; Gupta and Chaphalkar, 2015).

\section{CD14 monocyte surface marker}

Similarly, PBMC were cultured with variable concentration of aqueous leaves extract of Acacia catechu along with or without NDV (1:80 dilution, $10 \mu \mathrm{L})$ for $48 \mathrm{~h}$ in 96 -well plates. After incubation, treated and non treated PBMC samples of Acacia catech $u$ were collected and stained with CD14 FITC $(3 \mu \mathrm{L})$ monoclonal antibody. The samples were then incubated (at room temperature), lysed (red cell lysis buffer containing ammonium chloride, potassium bicarbonate and EDTA) and washed with PBS $(\mathrm{pH}, 7.2)$. The resulting stained cell pellet was resuspended in $500 \mu \mathrm{L}$ of PBS and run on a FACS Calibur flow cytometer (Gupta et al., 2015).

\section{Statistical analysis}

All values were mentioned as Mean \pm S.E. Data was represented by one-way ANOVA test (Boniferroni multiple comparison test).

\section{RESULTS AND DISCUSSION Cytotoxicity and PBMC proliferation (NDV) assay}

The effect of variable doses of aqueous leaves extract of Acacia catechu on human PBMC proliferation assay with NDV and also determined its cytotoxicity in absence of NDV containing aqueous leaves extract on PBMC 
using MT'T (Figure 1). For cytotoxicity based studies, aqueous leaves extract showed a dosedependent decrease in PBMC population at higher doses as compared to control where as PBMC cultured along with NDV containing variable concentration of aqueous leaves extract showed enhancement in proliferation at lower doses but there is significantly decline in NDV proliferation at higher doses as compared to control. In addition, NDV showed a significant enhancement of PBMC proliferation as compared to control. Overall, the data represents that Acacia catechu at higher doses showed cytotoxic as well as anti-viral activity at higher doses.

\section{CD14 monocyte surface marker}

The effect of aqueous leaves extract of CD14 monocyte surface marker on PBMC with or without NDV (Figure 2). There was a significant decrease in CD14 monocyte surface marker at higher doses of Acacia catechu against NDV as compared to control.

The present study describes about the importance of aqueous leaves extract of Acacia catech $u$ and showed its safe and effective antiviral agent against NDV. Normally, the virus cannot be able to mutate with the medicinal plants because of the complexity of chemicals e.g. Catechin, epicatechin, alkaloids, quercetin etc in the form of secondary metabolites that are present abundantly. As per the literature, medicinal plant i.e. Acacia catechu reported number of secondary metabolites e.g. terpenoids, flavonoids, phenolics etc that are present and showed its importance to provide potentially new chemotherapeutic agents against various dreadful pathogens.

According to Ayurveda, lot of research work has already been done related to immunopharmacological studies of medicinal plants with respect to humoral and cell mediated immune response using non specific antigen. These studies are of great importance for many immunopharmacologists pertaining to anti-viral, anti-inflammatory, anti-diabetic studies etc (Haider et al., 2011, Gupta et al., 2014). For these studies, our group used human PBMC (monocytes) which showed morphological heterogeneity, such as variability of size including granularity and nuclear morphology and determined its cytotoxicity and CD14 monocyte surface marker using with or without NDV. Firstly, cytotoxicity assay are essential for determining the responses of human $\mathrm{PBMC}$ in presence or absence of specific antigen i.e. NDV. It is already established that exposure of test candidates on human PBMC triggers a permanent growth arrest through the process of accelerated senescence in a fraction of the cell population (Gupta et al., 2015).

In short, loss of cellular viability measured through caliometric based assay i.e. MTT emerged as a favored method for assessment of cytotoxicity (Gupta et al., 2006).

One of the parameters interconnected with our immune system i.e. CD14 monocyte surface marker which is determined through flow cytometric analysis and provides reliable information whether aqueous extract of Acacia catech $u$ showed stimulatory or suppressive effect. Normally, viral pathogens in human increased the number of monocytes count in the form of CD14 (receptor for lipopolysaccharide) surface marker. Finally, flow cytometric results suggested that production of CD14 surface marker may depend on cell types (monocytes or macrophages) and their species origin, different cells having obviously individual necessity for signal transduction pathways. In particular, determination of CD14 marker from human PBMC assumed to play a considerable role in the pathophysiology of hormonal immune system. The findings of these preliminary studies showed that the aqueous extract of Acacia catechu against NDV virus and the results of anti-viral activity including its cytotoxic effect against NDV are shown in respective figures. The results indicate that aqueous leaves extract of Acacia catechu shows a dosagedependent correlation and found that aqueous leaves extract could significantly reduce the CD14 count in human PBMC exposed to NDV. The results of our immunopharmacological studies on human PBMC after exposing with NDV suggest that the aqueous leaves extract shows anti-viral effect on human PBMC.

These studies suggest that aqueous leaves extract of Acacia catechu significantly inhibits proliferation assay and $\mathrm{CD} 14$ monocyte surface marker when using NDV. 


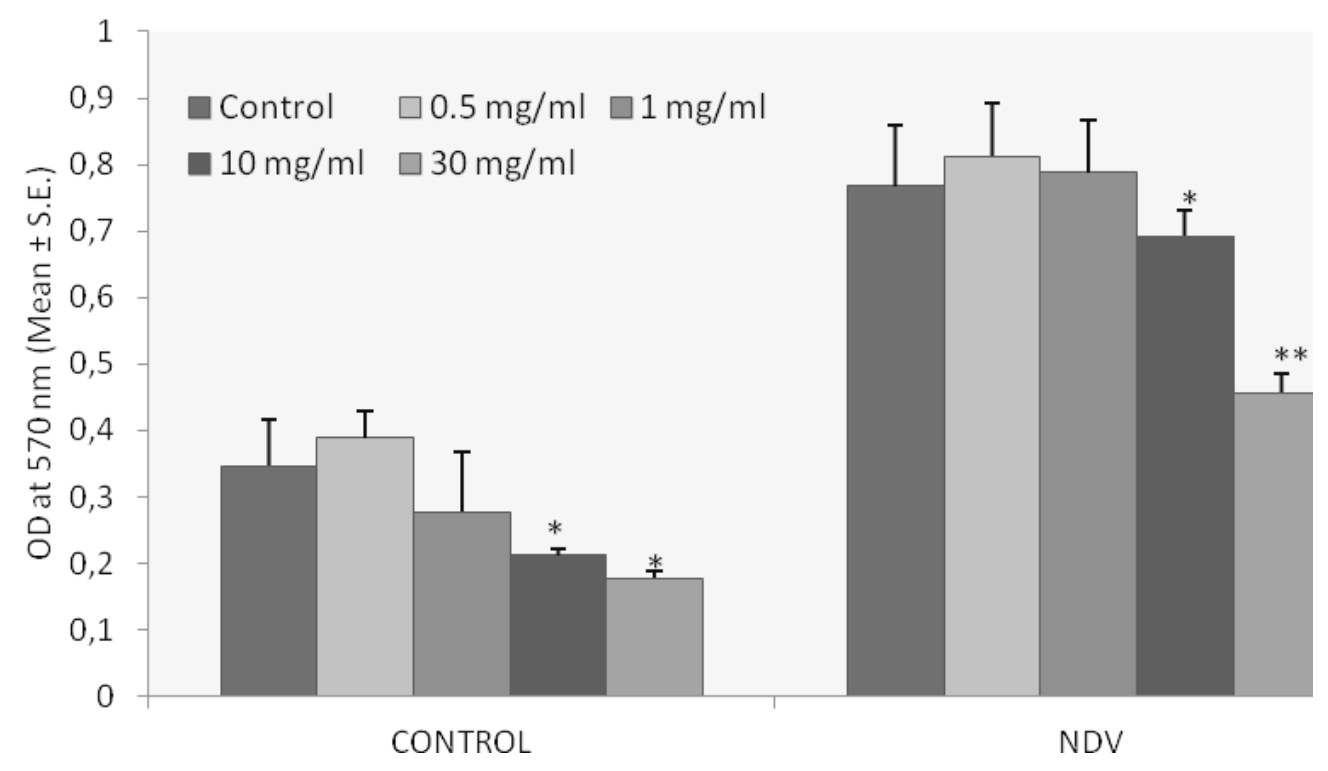

Figure 1. Effect of variable doses of aqueous leaves extract of Acacia catechu on NDV in human PBMC. PBMC were cultured with or without optimized dose of NDV along with variable doses of aqueous root extract $(0.5,1,10$ and $30 \mathrm{mg} / \mathrm{mL}, 50 \mu \mathrm{L})$ or NDV alone. After 3 days, proliferation was measured by MTT assay. The results are presented as Mean \pm S.E. $P$ values: $* P<0.05$, **P $<$ 0.01 , ***P $<0.001$ as compared to control.

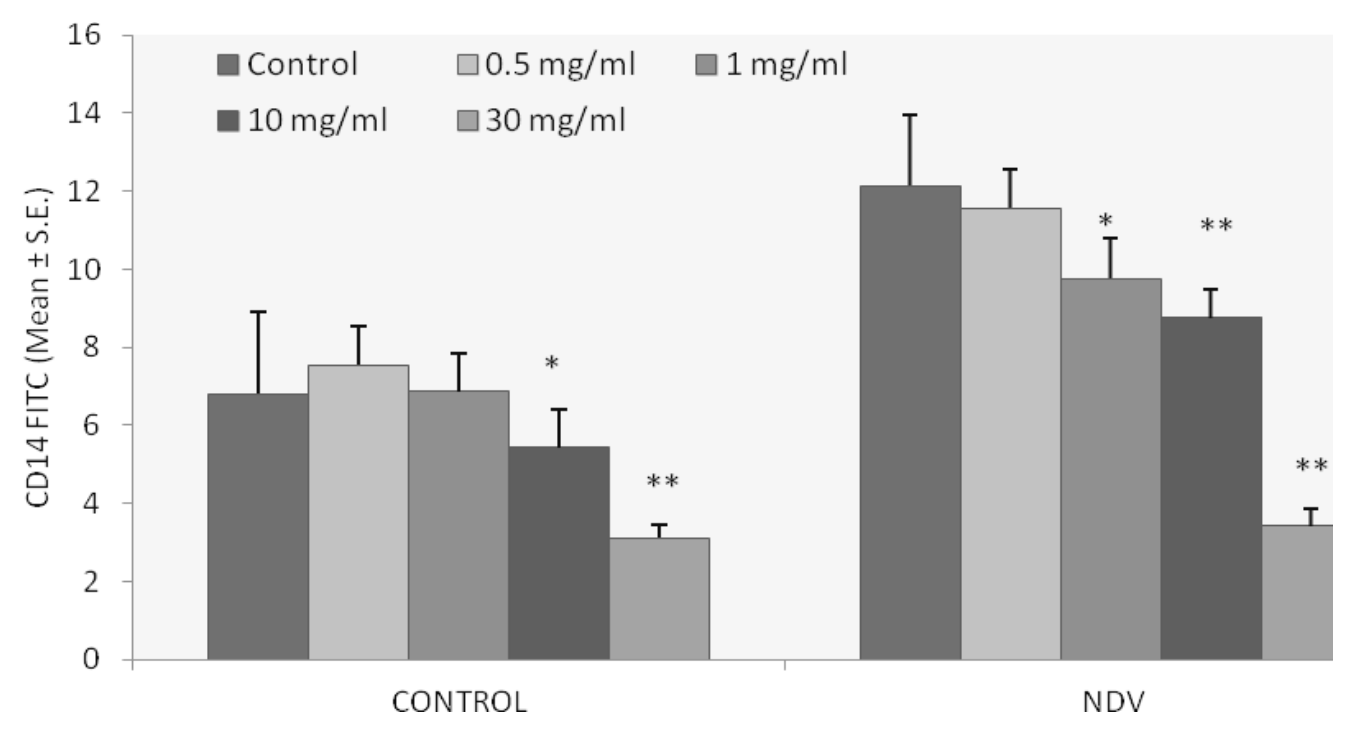

Figure 2. Flow cytometric analysis of aqueous leaves extract extracted from Acacia catechu on CD14 monocyte surface marker. PBMC were treated with variable doses of aqueous leaves extract $(0.5,1$, 10 and $30 \mathrm{mg} / \mathrm{mL}, 50 \mu \mathrm{L}$ ) along with or without NDV and then lysed and wash the cells with phosphate buffered saline and analyzed through flow cytometer (FACS, Calibur) using forward and side scatter gating applied for data acquisition of 10000 events of cell populations representing different phenotypes analyzed using cell quest software. 
Further investigations of the aqueous leaves extract should be done through in vivo assessment for immunopharmacological (antiviral) studies in mice models with identification of the major active candidates responsible for anti-microbial activities.

\section{ACKNOWLEDGEMENT}

We would also like to thanks Dr $\mathrm{H}$. Pawar Veterinary Doctor for collection of NDV samples under the project of BiovillageSustainable development through Biotechnology. And this studies were done under the programme of Biovillage scheme, Vidya Pratishthan's School of Biotechnology.

\section{REFERENCES}

Gupta A., Khamkar PR. and Chaphalkar SR. Review on medicinal plants to target and inhibit the epidermal growth factor receptor signaling in cancer and tissue repair therapy. Int. J. Advances in Pharmacy, Biology and Chemistry. 2014; 3(1): 210- 213.

Maciel MAM., Pinto AC., Veiga Jr. VF., Grynberg NF., Echevarria A. Medicinal plants: the need for multidisciplinary scientific studies. Quim Nova 2002; 25(3):429-438.

Maithani A., Versha P., Geeta P., Kumar D., Dhulia I. Pharmacognostical standardization of leaves of Azadirachta IndicaAn effective panacea for all disease. Int J Res Pharm Sci 2011; 2(4): 563 - 568.

Gupta A., Khamkar PR. and Chaphalkar SR. Anti-inflammatory activity of aqueous extract of Mimusops elengi on human whole blood and peripheral blood mononuclear cells. Int. J.Current trends in Pharm. Res.2014; 2: 638 - 645.

Patil SM. and Saini R. Antimicrobial activity of flower extract of Calotropis gigantea. Int. I Pharm Phytopharmacological Research 2014; 1 (4): 142-145.

Gupta A., Jagtap RB. and Chaphalkar SR. Antiviral activity of Azadirachta indica leaves against Newcastle disease virus: A study by in vitro and in vivo immunological approach. Int. J.Current Trends in Pharmacentical research 2014; 2: 494 - 501.
Lakshmi T. and Rajendran R. Estimation of biofavonoid quercetin in Acacia catechu ethanolic leaf extract by HPLC method. Int. J.Pharma and Biosciences 2012; 3 (1): $171-178$.

Ray D., Sharatchandra K. and Thokchom I. Antipyretic, antidiarrhoeal, hypoglycaemic and hepatoprotective activities of ethyl acetate extract of Acacia catechu wild. In albino rats. Ind $J$ of pharmacology 2006; 38(6):408-13

Guleria S., Tiku A., Singh G., Vyas D and Bhardwaj A. Antioxidant Activity and Protective Effect Against Plasmid DNA Strand Scission of Leaf, Bark, and Heartwood Extracts from Acacia catechu. J of Food Science 2011; 76(7): 959-64.

Gupta A. and Chaphalkar SR. Immunopharmacological screening of aqueous root extract of Santalum album. Journal of Herb Med Pharmacology 2016; 5 (1):07 - 11.

Gupta A. and Chaphalkar SR. Potential immunosuppressive and antiinflammatory activity of aqueous extract of Mangifera indica. Advanced herbal medicine 2015; 1(4): 47 - 54.

Gupta A and Chaphalkar SR. Inhibitory potential of aqueous leaves extract of Messua ferrea and Mimusops elengi on antigen specific immune response using human whole blood. Asian Journal of medical and Pharmaceutical researches 2015, 5 (3): $22-26$.

Haider S., Nazreen S., Alam MM., Gupta A., Hamid H. and Alam MS. Antiinflammatory and anti-nociceptive activities of ethanolic extract and its various fractions from Adiantum capillus veneris Linn. Journal of Ethnopharmacology 2011; 138 (3): 741-747.

Gupta A. and Chaphalkar SR. Immunorestorative and anti-inflammatory activity of leaf aqueous extract of Calotropis gigantean using flow cytometry. International Journal of drug discovery and herbal research 2014; 4(4): 761 - 765. 
Gupta A. and Chaphalkar SR. Immunopharmacological activity of Zingiber officinale on human peripheral blood mononuclear cells. Asian Journal of Medical and Pharmacentical researches 2015; 5(2): 13 17.
Gupta A., Khajuria A., Singh J, Bedi KL., Satti NK., Dutt P., Suri KA., Suri OP and Qazi GN. Immunomodulatory activity of biopolymeric fraction RLJ-NE-205 from Picrorbiza kurroa. Int. Immunopharm. 2006; 6 (10): 1543 - 1549. 